\title{
EL PARTO VAGINAL EN PACIENTE CON ANTECEDENTE DE CESAREA
}

\author{
Doctor José Gabriel Acuña Díaz
}

A pesar de los numerosos estudios publicados sobre el tema, respaldados en largas series de casos, existen aún divergencias sobre la conducta a seguir en la paciente con antecedente de cesárea. Por esta razón emprendimos en el Instituto Materno Infantil Concepción Villaveces de Acosta una revisión del tema. Presentamos los resultados obtenidos en los casos estudiados en un lapso de 19 meses, comprendido entre el mes de septiembre de 1962 y el mes de marzo de 1964.

Tres criterios continúan enfrentados acerca del problema.

1. El grupo que sostiene que una cesárea debe ser seguida siempre de cesárea. Insiste en el peligro que la cicatriz uterina representa para los partos posteriores.

2. Un segundo grupo permite el parto vaginal en ciertos casos, considerándolo peligroso si la intervención previa fue clásica, cuando se ha presentado infección uterina postoperatoria, cuando la cesárea anterior fue por placenta previa, en los casos de sobredistensión uterina por hidramnios o ges- tación múltiple, en caso de comprobación de cicatriz uterina defectuosa y si coexisten anomalías uterinas.

3. El último grupo permite el trabajo de parto en todos los casos en que no persista la indicación anterior.

Nosotros creemos que la conducta, naturalmente, ha de ser de selección por valoración de los datos obtenidos en la evaluación del caso, tanto desde el punto de vista quirúrgico (técnica, cirujano, medio hospitalario, etc.), como obstétrico, es decir, el que hace relación a las condiciones del caso que se estudia. Igualmente consideramos que tiene enorme importancia la evaluación de la cicatriz, cuyo estudio debe intensificarse.

A manera de información analizaremos los datos bibliográficos más importantes que hemos recopilado:

Riva y Teich (1) presentan un estudio de 214 pacientes con antecedentes de cesárea, de las cuales 56 (26.4\%), fueron intervenidas nuevamente y 158 tuvieron parto vaginal (73.6\%); de las reintervenidas 36 fueron extraperitoneales, 12 segmentarias y 4 clásicas (dos 
con histerectomía total y dos con subtotal); en los 158 partos vaginales se aplicaron 121 fórceps bajos y 4 en estrecho medio. Hubo 29 partos espontáneos en vértice y 4 en presentación de pelvis. En ninguno de los partos vaginales se presentó ruptura uterina.

Reyes Ceja (2) sometió a prueba de trabajo 190 pacientes con antecedentes de cesárea, prueba consistente en amniotomía aproximadamente en $4 \mathrm{cms}$. de dilatación, administración de antiespasmódicos, refuerzo de trabajo con soluciones diluídas de Pitocin en los casos indicados y control de la respuesta cervical, la progresión del encajamiento y la vitalidad fetal. Practicó únicamente 4 cesáreas de repetición, todas por sufrimiento fetal; atendió 118 casos espontáneos en presentación cefálica de vértice y 4 en presentación de pelvis; aplicó 65 fórceps y practicó una versión y una embriotomía. No presenta rupturas uterinas. Somos enemigos de la práctica de la versión y la embriotomía en las pacientes con cicatriz uterina de cualquier naturaleza, las cuales nos parecen francamente peligrosas.

Aguiero, Marcano y Páez (3, 4, 5) en el estudio de 629 pacientes que tuvieron partos posteriores a una cesárea, encontraron que $254(42 \%)$ fueron por vía vaginal, 321 (53\%) fueron por cesárea y 29 (4.8) fueron tanto por cesárea como por vía vaginal. Su estudio es global referido a la historia obstétrica completa de pacientes intervenidas en la "Maternidad Concepción Palacios", de Caracas y atendidas en uno o más de sus partos posteriores en la misma institución; presentan 21 ca- sos de dehiscencia de la cicatriz uterina $(3.3 \%)$ y hacen consideraciones detenidas sobre los factores predisponentes y causantes, tales como: técnica quirúrgica, hemostasis, postoperatorio, paridad, número de intervenciones, trabajo de parto, sitio de inserción placentaria, etc.

Donaldson, Lamkee y Alvarez (7), revisan la conducta en el manejo de la operación cesárea en dos hospitales, universitario y particular, afirmando que en los casos con antecedente de cesárea, ésta no necesariamente significa nueva cesárea. Añaden, sin embargo, que en tratándose de pacientes particulares la tendencia en su medio en estos casos es practicar la cesárea de repetición. Esta "doble conducta" está en contradicción con la práctica usual en nuestro medio y con la de la escuela a la cual pertenecemos.

Jesurun, citado por Peralta Cayón (9), en un estudio de 222 pacientes con antecedentes de cesárea, encuentra 109 cesáreas de repetición $(49 \%)$ y 113 partos por vía vaginal $(51 \%)$, de los cuales $104(95 \%)$ fueron intervenidos con fórceps bajo; usó el Pitocin en 6 casos; presenta 5 rupturas uterinas.

Del Corral y Muñoz (8) en su trabajo "Evaluación clínica para el parto después de cesárea", presentan 125 casos de los cuales 63 terminaron por vía vaginal (31 partos espontáneos y 32 con fórceps) y 62 fueron intervenidos nuevamente. La incidencia de ruptura fue de 6 casos, sin muerte materna. Consideran que un estudio cuidadoso de cada caso permite la oportunidad de parto vaginal aproximadamente a la 
mitad de las pacientes y que la cesárea de repetición no evita completamente el peligro de ruptura uterina.

Peralta Cayón (9) presenta un estudio de 42 pacientes con antecedente de cesárea atendidas en una o más gestaciones posteriores a la misma, para un total de 65 partos y un aborto. De las 41 pacientes atendidas en su parto en la primera gestación postcesárea, 21 fueron reintervenidas $(51.9 \%)$ y 20 tuvieron parto por vía vaginal (48.1\%); 32 casos tenían antecedente de una cesárea, 8 de dos y 1 de tres. En todos los casos se aplicaron soluciones de Pitocin i. v., asociado a gangliopléjicos, antiespasmódicos o sedantes, para inducción, estimulación o conducción del trabajo. No presenta incidencia de ruptura uterina.

Cárdenas y Zornosa (10), estudiando la cesárea iterativa, presentan 100 casos de pacientes, de las cuales 60 tuvieron parto por vía vaginal $(60 \%)$, 49 espontáneos y 11 con fórceps; 38 fueron reintervenidas por su antecedente de cesárea $(38 \%), 15$ de ellas con prueba de trabajo, y 2 terminaron en aborto $(2 \%)$; presentan un caso de ruptura segmentaria incompleta sin mortalidad materna ni fetal.

Sanz (11) sobre 135 casos del Instituto Materno Infantil, presenta 52 partos espontáneos, 21 intervenidos con fórceps, 1 extracción podálica y solamente 56 cesáreas de repetición; los 5 casos restantes corresponden a rupturas uterinas, con una muerte materna. En su opinión esta complicación es debida al abandono del trabajo de parto o a la práctica de maniobras obstétricas peligrosas en pacientes con antecedente de cicatriz uterina. Llama la atención sobre la frecuencia de la distocia de contracción por defecto en pacientes con antecedente de cesárea.

Castelazo Ayala (12) permite el trabajo de parto bajo condiciones especiales y sostiene que la conducta final es variable porque las normas dependen de numerosas circunstancias, locativas e individuales, que impiden cumplirlas con exactitud; menciona los resultados obtenidos por Reyes Ceja, antes enumerados, pertenecientes a la maternidad a su cargo.

En oposición a las experiencias y opiniones anteriores, Bak y Hayden, citados por Del Corral y Muñoz (8) después de estudiar 52 casos de ruptura uterina en 71.473 partos en Chicago Lying in Hospital, establecen en forma segura que ellos creen que después de una cesárea debe practicarse siempre cesárea. Donald y Frazer en Inglaterra, expresaron en 1953, que la cesárea de repetición está muy lejos de ser la regla aceptada o practicada en Inglaterra.

Pillsbury (13) piensa que el aforismo "una cesárea, siempre cesárea" conserva aún su vigencia; permite el parto vaginal después de cesárea solo en circunstancias excepcionales con facilidades quirúrgicas a disposición inmediata. Presenta un estudio de 2.316 cesáreas sin mortalidad materna, insistiendo en la importancia de ciertos factores adicionales como junta médica por especialistas, banco de sangre, anestesia, etc., para disminuír las complicaciones.

Presentamos (14) hace dos años y medio una revisión estadística de los resultados obtenidos en el Instituto Ma- 
terno Infantil de Bogotá en 221 casos de pacientes con antecedentes de cesárea, de los cuales $124(56 \%)$ tuvieron parto por vía vaginal y $97(44 \%)$ fueron reintervenidos por diversas causas; anotamos que se permitió el trabajo especialmente en los casos con antecedentes de partos previos por vía vaginal, aunque intervenidos más de una vez, y en aquellos que por ingresar en estado avanzado del parto, sin sintomatología de complicación dependiente de la cicatriz, permitieron una espera adecuada bajo estricta vigilancia; la incidencia de ruptura alcanzó al $1.3 \%$ ( 3 casos) y no hubo mortalidad materna.

De acuerdo a la revisión anterior, creemos que a excepción de algunas escuelas americanas, que reintervienen un elevado porcentaje de casos y de otras, poco numerosas, que permiten el parto vaginal a gran número de pacientes, la conducta se encuentra en un justo medio, aceptado por varias escuelas, cuyos resultados concuerdan ampliamente con los obtenidos por nosotros.

\section{PLAN DE TRABAJO}

Después de un estudio detenido rel tema establecimos una norma de trabajo dividiendo las pacientes en grupos de acuerdo a sus antecedentes y a las características de los datos obteni los en el examen de ingreso. El estudio comenzó en la consulta prenatal, en los contados casos en que las pacientes asistieron a ella, para continuar con la enferma hospitalizada. Consideramos que si bien es cierto que muchas de ellas pueden dar a luz por vías natu- rales, no es menos cierto que afrontan en ocasiones riesgos innecesarios que ponen en peligro su vida y la del feto. Practicamos rutinariamente u $\mathrm{n}$ a anamnesis cuidadosa, insistiendo en el aspecto quirúrgico y los antecedentes obstétricos, además de un cuidadoso examen y valoración del embarazo actual; no fue posible el estudio de la cicatriz ya que el elevado número de casos atendidos, la escasez de personal y de recursos, y por encima de todo, la falta de cooperación e interés del personal que asiste al Instituto, fueron motivos insalvables.

Los puntos principalmente considerados fueron los siguientes:

1. Valoración de los antecedentes:

Indicación de la intervención.

Número de intervenciones.

Técnica o tipo de cesárea practicado. Complicaciones operatorias y postoperatorias.

Estudio cronológico de partos vaginales e intervenidos y abortos.

Fecha de intervención y lugar.

Cirujano que intervino.

Edad gestacional al ser intervenida, número de fetos.

Estado de las membranas, dilatación cervical, horas de trabajo.

Otros datos (hemorragia ,transfusión, infección, etc.).

\section{Valoración obstétrica:}

Fecha de la últ i m a menstruación, edad gestacional.

Estado fetal, número de fetos. 
Tamaño fetal, presentación, altura de la presentación y caracteres.

Presencia de distensión, c a n t i d a d aproximada de líquido amniótico.

Parto: iniciación, horas de trabajo, características del mismo.

Estado del cuello, dilatación, estado de las membranas, líquido.

Presencia de hemorragia genital y dolor hipogástrico.

Pelvigrafía y clasificación de la pelvis.

Patología general u obstetricia, asociadas.

De acuerdo con la valoración de estos datos establecimos en cada caso un pronóstico, favorable o desfavorable para parto vaginal; en el primer caso se permitió la prueba de trabajo (corta, media o larga) y en el segundo se intervino poco tiempo después del ingreso de la paciente.

Se establecieron tres grupos:

1. Cesárea de repetición por indicación absoluta:

Por persistencia de la indicación.

Por aparición de nueva indicación.

Por antecedente de cesárea atípica, clásica o complicada.

En secundigestante "añosa" con antecedente de cesárea.

En caso de presentaciones paraeutócicas (cara, bregma, pelvis).

En pacientes con antecedentes de 2 o más cesáreas previas.
Cuando la cesárea anterior la practicó médico no especializado y en medio inadecuado.

En caso de sobredistensión uterina.

En caso de distosia de contracción por exceso.

En pacientes con patología de partes blandas asociadas (fístulas).

2. Cesárea de repetición, de indicación relativa:

En caso de antecedente de infección uterina postoperatoria

En caso de infección amniótica, con feto vivo o muerto.

En caso de feto muerto preparto.

En caso de ruptura prematura de membrana, sin complicaciones.

En caso de inserción baja, no previa, de placenta.

\section{Parto vaginal:}

Se permitió el trabajo de pario en las pacientes que se apartaban de las indicaciones anteriores, especialmente en los casos de antecedentes de partos espontáneos previos.

La prueba de trabajo consistió en la amniotomía una vez alcanzados los 4 cms., de dilatación, sedantes y coordinadores de la contracción uterina; se valoraron para control y conducción, la dinámica uterina, la respuesta cervical (borramiento, dilatación, consistencia), la duración del trabajo, el encajamiento (progresión) y la vitalidad fetal.

La prueba de trabajo se suspendió en los casos de distensión del segmento o inminencia de ruptura uterina, su- 
frimiento fetal y en caso de prueba negativa (falta de progresión de la presentación y la dilatación cervical con buena dinámica uterina y canal pelviano dentro de límites normales).

Se consideró peligroso el empleo de los ocitócicos y sólo se permitió en casos especiales bajo control personal del Jefe de Clínica; se contraindicaron el trabajo prolongado (más de 12 horas), la maniobra de Kristeller o presión sobre el fondo uterino para lograr el desprendimiento, las maniobras intrauterinas (versión, mutación) y las intervenciones laboriosas con fórceps, especialmente las de rotación.

Fueron indicaciones de rutina la aplicación de fórceps bajo o de ventosa de Malmstrom y la revisión uterina.

Al igual que Eastman, citado por Cárdenas y Zornosa (10), creemos que el cuidado prenatal de estas pacientes debe ser orientado de manera especial; es obligatorio formarse un concepto de las posibilidades de cada pa- ciente de acuerdo con los antecedentes y datos del examen; cuando se impone la cesárea iterativa debe insistirse en precisar la aparición de los movimientos fetales y de la fetocardia, criterio útil posteriormente para valorar la madurez fetal. La palpación del segmento en busca de signos anormales de sensibilidad a nivel de la cicatriz debe ser rutinaria. La paciente debe hospitalizarse tan pronto como aprecie las primeras contracciones. En los casos de cesárea programada, iterativa, una vez cumpla la $38^{\text {a }}$ semana.

\section{MATERIAL}

Estudiamos 614 casos de pacientes con antecedentes de cesárea los cuales agrupamos en favorables y desfavorables de acuerdo con sus antecedentes quirúrgicos y obstétricos y con la valoración de cada caso en el momento de su ingreso al Instituto, según las normas antes mencionadas.

\section{Casos}

Favorables

Desfavorables

Partos vaginales

Cesáreas

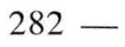

332

259

355
Parto vaginal

Nueva cesárea

Parto vaginal

Nueva cesárea

Favorables

Desfavorables

Favorables

Desfavorables

252
30
7
325
272
7
30
335

89.4

10.6

2.1

97.9

272 
I. Mencionaremos inicialmente el vención, ceñidas en su gran mayoría a grupo de pacientes considerado desfa- los esquemas previstos para las pacienvorable para parto vaginal, el cual se- tes con antecedente de cirugía uterina rá motivo de una publicación posterior, (cuadro 1).

y las causas que motivaron la reinter-

\begin{tabular}{lrc}
\hline Indicación & Principal & Relativa \\
\hline Estrechez pélvica & 140 & 15 \\
Dos o más cesáreas previas & 57 & 2 \\
Situación transversa & 22 & 3 \\
Sufrimiento fetal & 17 & 11 \\
Presentación de pelvis & 17 & 3 \\
Placenta previa a & 15 & 2 \\
Secundigestante "añosa" con cesárea previa & 14 & 2 \\
Cesárea en medio inadecuado, & 7 & \\
Cirujano desconocido & 5 & \\
Distención uterina (gemelar, hidramnios) & 5 & \\
Falta de encajamiento & 5 & 4 \\
Antecedente de fístulia vesicovaginal & 4 & \\
Distocia de contracción por defecto & 3 & \\
Situación oblicua & 3 & \\
Desproporción cefalo-pélvica & & \\
Otros: abruptio, frente, ruptura prematura & & \\
de membranas e infección amniótica (2 & & \\
c/u.); inminencia de ruptura, polisisto- & & \\
$\quad$ lia, distocia cervical, ruptura uterina y & & \\
$\quad$ procidencia de miembro superior (1 c/u. & 11 & \\
\hline
\end{tabular}

Siete casos considerados desfavorables a su ingreso al Instituto terminaron en parto vaginal a causa de la demora en la práctica de la intervención programada, ocasionada ésta por diversos motivos; 3 de ellos con presentaciones de pelvis, 2 con antecedente de dos cesáreas y 2 , finalmente, con estrechez relativa de la pelvis. Todos ingresaron en trabajo de parto; 5 de ellos con 5 o más cms., de dilatación; en ninguno se presentó ruptura uteri- na a pesar de que en uno de los casos de presentación de pelvis se aplicó un refuerzo de la contracción con 2 unidades de Pitocin en $500 \mathrm{cc}$ de Dextrosa al $5 \%$ en agua destilada.

II. Encontramos 282 casos considerados favorables para el parto vaginal, de los cuales 252 (89.4) tuvieron parto de acuerdo a la valoración y 30 (10.6 por ciento) fueron intervenidos nuevamente por cesárea, por los motivos 
que más adelante anotaremos; estos 252 ble, dan un total de 259 partos vagicasos, agregados a los 7 anteriormente nales, cuya indicación anterior fue la mencionados de valoración desfavora- siguiente (cuadro 2):

\begin{tabular}{|c|c|c|c|}
\hline Indicación & Nuliparas & $\begin{array}{l}\text { Cesárea } \\
\text { Ult. Parto }\end{array}$ & $\begin{array}{c}\text { Parto } \\
\text { Post Ces. }\end{array}$ \\
\hline Situación transversa & 6 & 12 & 16 \\
\hline Desproporción cefalopélvica & 4 & 2 & 15 \\
\hline Procindencia del cordón & 4 & 11 & 7 \\
\hline Presentaciones de frente y cara & 4 & 1 & 1 \\
\hline Sufrimiento fetal & 11 & 6 & 9 \\
\hline Placenta previa & 1 & 17 & 20 \\
\hline Distocia cervical & 10 & 1 & 1 \\
\hline Infección amniótica & 2 & - & 1 \\
\hline Presentación de pelvis & 2 & 1 & 3 \\
\hline Estrechez péliva & 4 & - & 6 \\
\hline Desprendimiento prematuro de placenta & 5 & 3 & 1 \\
\hline Distocia de partes blandas & - & 3 & 1 \\
\hline Embarazo gemelar & - & 1 & 1 \\
\hline Condilomatosis genital & 2 & - & - \\
\hline Trabajo prolongado & 1 & - & 3 \\
\hline Muerte neonatal & - & 1 & 1 \\
\hline Procindencia de mano & - & 1 & - \\
\hline Herida abdominal cortopunzante & 1 & - & 1 \\
\hline Embarazo prolongado & - & - & 1 \\
\hline Primigestante añosa & 2 & - & 1 \\
\hline Ruptura uterina & - & - & 1 \\
\hline Eclampsia & - & - & 4 \\
\hline Sin dato de indicación & 5 & 3 & 33 \\
\hline TOTAL & 61 & 63 & 135 \\
\hline
\end{tabular}

Dividimos los casos en tres grupos: nulíparas, multigestantes con cesárea en su último parto y multigestantes con uno o más partos posteriores a la cesárea practicada. Muy claramente lo mencionaron Del Corral y Muñoz (8) en el primer grupo se desconoce la ca- pacidad pélvica de la paciente. En el segundo se deben vagilar de manera especial las condiciones de la cicatriz y el aspecto quirúrgico, ya que los partos anteriores permiten suponer una pelvis de buena capacidad, en caso de que sus niños hayan sido de tamaño 
normal. Las pertenecientes al último grupo han puesto ya a prueba la bondad de su cicatriz y representan naturalmente el menor riesgo, aunque esto no significa que no requieran vigilancia.

Dentro de este grupo se encuentran 17 casos con antecedentes especiales, así: 15 casos con dos cesáreas previas, uno con tres y uno con antecedente de laparatomía, posterior a ruptura uterina durante la práctica de embriotomía raquídea. Por la dificultad para conseguir las historias clínicas anteriores en el momento del ingreso de la paciente, pues ingresan generalmente en trabajo de parto avanzado, no se poseen datos suficientes para modificar la conducta, que sería en ocasiones la repetición de la cesárea.

En 30 casos considerados favorables para parto vaginal hubo de praeticarse nuevamente la cesárea. Los dividimos para su estudio en dos grupos, al primero de los cuales corresponden 12 casos de valoración adecuada, y al segundo 18 casos, en los que la valoración fue inadecuada.

Valoración adecuada:

Cinco fueron intervenidas por sufrimiento fetal, asociados en dos casos a distocia de contracción por defecto, en dos a distocia cervical y en el restante a infección amniótica.

Cuatro por distocia de contracción por defecto, en tres asociada a distocia cervical; uno de estos casos fue tratado con solución de Pitocin intravenosa, sin resultado satisfactorio.

No figura la causa de intervención en los tres casos restantes.
Valoración inadecuada:

En los 18 casos de valoración inadecuada se encontraron los siguientes datos adicionales: en seis casos estrechez relativa de la pelvis, uno asociado a distocia de contracción por defecto y uno a sufrimiento fetal.

En 2 casos antecedentes quirúrgicos desconocidos y medio hospitalario no recomendable, uno en secundigestante "añosa" con antecedente de cesárea y otro con sufrimiento fetal e inminencia de ruptura uterina.

Tres casos con desproporción cefalopélvica; uno en paciente con presentación de vértice en variedad posterior, asinclítica, con feto muerto por procidencia de cordón, en el cual se hizo un intento de ventosa de Malmstrom, fallido, antes de ser intervenido; el segundo en paciente con feto grande que terminó en ruptura uterina por falta del control del trabajo en el Servicio; el último en paciente con feto grande $\mathrm{e}$ hidramnios.

El resto de los casos presentaban: placenta previa marginal uno, hipermadurez con historia de muerte neonatal uno, eventración e infección amniótica uno, dos cesáreas previas dos, sufrimiento fetal al ingreso uno y distocia de contracción por defecto uno.

\section{TRABAJO DE PARTO}

Comentamos en nuestra comunicación anterior (14) que cerca del $92 \%$ de las pacientes estudiadas regresó en trabajo de parto y sólo escaso número de ellas asistió a la consulta prenatal; desafortunadamente esta situación no se 
ha modificado y encontramos, en los 259 casos estudiados, los siguientes datos de dilatación cervical al examen de ingreso (cuadro 3):

\begin{tabular}{|c|c|c|}
\hline Dilatación & Casos & $\%$ \\
\hline Menos de $3 \mathrm{cms}$ & 61 & 23.5 \\
\hline De 4 a $6 \mathrm{cms}$ & 89 & 39.4 \\
\hline De 7 a $9 \mathrm{cms}$ & 59 & 22.8 \\
\hline Dilatación completa & 22 & 8.4 \\
\hline Sin trabajo & 12 & 4.6 \\
\hline Sin dato & 6 & 2.3 \\
\hline TOTAL & 259 & 100.0 \\
\hline
\end{tabular}

Esto ha inferido en la conducta $y$ resultados finales, porque es seguro que algunos de los casos ingresados con trabajo de parto avanzado hubieran sido programados para la cesárea de repetición; esperamos que el incremento e importancia que desde hace dos años se ha dado a la consulta prenatal en el Instituto influya favorablemente, con el correr del tiempo, en una mayor vinculación de estas pacientes al servicio.

Encontramos un caso de polisistolia. Por el contrario, las distocias de contracción por defecto fueron numerosas, de acuerdo con los datos siguientes:
Contracciones de intensidad baja

Contracciones espaciadas

Contracciones irregulares, cortas

y de baja intensidad

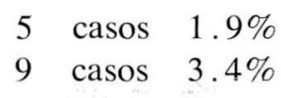

67 casos $25.8 \%$
Acerca de la irregularidad del trabajo en pacientes con antecedentes de cesárea Sánz (11), Del Corral y Muñoz (8) y Acuña Díaz (14), han mencionado que éste no se realiza regularmente en algunos casos, posiblemente porque la cicatriz se comporta como un obstáculo a la repartición de la onda contráctil; nosotros habíamos notado mayor incidencia de alteraciones de la contracción en las pacientes con más de una cesárea previa. Peralta Cayón (9) anota que las irregularidades contráctiles no son raras en las cesáreas previas.

Sobre la discutida posibilidad de 1 empleo de soluciones de Pitocin por vía intravenosa, más distantes aún se encuentran las distintas escuelas. Noso- tros empleamos el refuerzo de trabajo con soluciones diluídas en 4 casos, uno de ellos en paciente con presentación de pelvis, en dilatación completa (2 U); otro en paciente con distocia de contracción por defecto ( $2 \mathrm{U})$, suspendido una hora después de su aplicación al comprobarse antecedentes desconocidos; el tercero en un caso de paciente con 3 cesáreas anteriores, con aborto de 20 semanas en evolución (1 U); el último en paciente con antecedente de dos cesáreas, con feto muerto por procidencia del cordón, al cual se aplicó ventosa en estrecho medio y fórceps de desprendimiento, comprobándose después ruptura de la cicatriz uterina. Se aplicaron dos inducciones, la $1^{\text {ọ }}$ en paciente con embarazo hipermaduro y 
la segunda en caso de feto muerto en útero a las 30 semanas de gestación.

Jesurun, citado por Peralta (9), presenta seis casos del uso del Pitocin intravenoso.

Riva y Teich (1) siete casos. Del Corral y Muñoz (8) afirman que no se debe usar el Pitocin en las pacientes con antecedentes de cesárea.

Septién (16) dice que solamente contando con equipo para infusión exacta de soluciones de ocitócicos y de registro de las contracciones uterinas, se puede inducir a las pacientes con cesárea previa.

Castelazo ayala (12) dice que "emplea con frecuencia soluciones de ocitocina endovenosa en los casos de hiposistolia, siempre condicionando su efecto a los resultados obtenidos y buscando con ello producir una actividad uterina rigurosamente normal".

Entre nosotros la mayor experiencia corresponde a Peralta Cayón (9) quien ha practicado en 65 ocasiones, 42 pacientes, "inducciones, refuerzos, estimulaciones o conducciones de parto" usando simultáneamente gangliopléjicos, espasmolíticos o sedantes; persigue evitar la cesárea de repetición hasta donde sea posible, tratando al mismo tiempo de disminuír la elevada frecuencia de la intervención; hace detenidas consideraciones sobre los casos estudiados, dosis empleadas, técnica de aplicación, sensibilidad individual uterina al Pitocin, complicaciones, etc. No presenta casos de mortalidad materna ni fetal y tampoco rupturas uterinas. En los 41 casos de gestantes a término, o en período de viabilidad, tratadas con pitocin intravenoso, obtuvo 20 partos vaginales y repitió la intervención en 21 .

\section{P A R T O}

El parto vaginal en los 259 casos, dos gemelares, terminó tal como lo muestra el cuadro siguiente (cuadro 4).

\begin{tabular}{llrlr}
\hline Espontáneo: & Vértice & 124 & casos & $47.8 \%$ \\
& Pelvis & 8 & casos & $3.0 \%$ \\
Fórceps: & Directo & 70 & casos & $26.3 \%$ \\
& Rotación & 7 & casos & $2.6 \%$ \\
Fórceps y ventosa & 3 & casos & $1.4 \%$ \\
Ventosa de Malmstrom & 41 & casos & $15.9 \%$ \\
Espátulas de Thierry & 7 & casos & $2.6 \%$ \\
Espátulas y ventosa & 1 & casos & $0.4 \%$ \\
\hline
\end{tabular}

A pesar de ser nuestra norma auxiliar o intervenir el parto en su período final no fue posible hacerlo en buen número de casos; los motivos principales fueron los siguientes: exceso de tra- bajo (promedio de atendidas diariamente cerca de 60 casos), escasez de personal de anestesia, estado avanzado de trabajo de parto en las pacientes con una cesárea previa y partos vaginales 
previos o posteriores a la misma, falta de educación y cooperación del personal atendido en la maternidad.

Después del parto se procuró la revisión uterina; ésta fue completa en 126 casos $(48.6 \%)$, segmentaria en 65
(25.1\%); no se realizó en 68 de los casos atendidos $(26.3 \%)$.

La prueba de trabajo se consideró corta, media o larga, según se permitiera la evolución del parto cuatro, ocho, o doce horas. Los resultados fueron los siguientes (cuadro 5).

$\begin{array}{lrlc}\text { Trabajo de menos de } 4 \text { horas } & 40 & \text { casos } & 15.5 \% \\ \text { De } 4 \text { a } 8 \text { horas } & 112 & \text { casos } & 43.2 \% \\ \text { De } 8 \text { a } 12 \text { horas } & 68 & \text { casos } & 26.3 \% \\ \text { Más de } 12 \text { horas (prolongado) } & 32 & \text { casos } & 12.3 \% \\ \text { Sin dato } & 7 & \text { casos } & 2.7\end{array}$

En el total de casos con parto vaginal, 259 y los 30 considerados favorables, a los cuales se permitió la prueba de trabajo, tuvimos 4 casos de ruptura uterina, incompletas y 1 completa, para una incidencia de $1.5 \%$.

En 2 casos de dehiscencia de cicatriz segmentaria los fetos fueron de vitalidad normal y no requirieron intervención; el tercer caso corresponde a una paciente multípara con antecedente de dos cesáreas, con feto grande, muerto por procidencia del cordón, en la cual se aplicó un refuerzo de trabajo con una unidad de Pitocin, ventosa de Malmstrom en estrecho medio en 8 a 9 centímetros de dilatación y fórceps de desprendimiento; en la revisión se encontró ruptura de la cicatriz segmentaria, practicándose la laparatomía e histerorrafia. El último caso corresponde a una gestante con cesárea en su primer parto por desproporción ce- falo-pélvica, que permaneció cerca de 5 horas en 6 centímetros de dilatación, sin progresión en el encajamiento, sin control, y que presentó ruptura corporal longitudinal media; se practicó laparatomía e histerorrafia; la madre salió del Instituto en buenas condiciones.

Mencionamos inicialmente la incidencia de ruptura presentada por otros autores, razón por la cual no insistimos ahora en ella.

\section{RESULTADOS FETALES}

En relación con resultados fetales los datos son los siguientes, anotando que en términos generales las posibilidades de parto vaginal son inversamente proporcionales al peso del niño; tal el caso de la paciente con antecedentes de 3 cesáreas por estrechez pélvica que presentó aborto de 20 semanas, con feto de 600 gramos (único caso de aborto). (Cuadro 6). 


\section{Peso fetal:}

Menos de 100 gramos

$\begin{array}{rr}1 & 0.4 \% \\ 42 & 16.0 \% \\ 188 & 0.72 \% \\ 28 & 10.7 \% \\ 2 & 0.9 \% \\ 261 & \end{array}$

Hasta 2.500 gramos

De 2.501 a 3.500 gramos

Más de 3.501 gramos

Sin dato

TOTAL

261

\section{Edad:}

De 20 semanas

Menos de 36 semanas

$\begin{array}{cr}1 & 0.5 \% \\ 34 & 13.0 \% \\ 201 & 77.2 \\ 19 & 7.3 \% \\ 4 & 2.0 \%\end{array}$

De 37 a 40 semanas

Más de 40 semanas

Sin dato

TOTAL

259

\section{Apgar:}

Dos a seis

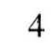

1. $52 \%$

Siete

Ocho

Nueve

Diez

194

Sin dato

Mortinatos

TOTAL

Los datos de morbilidad fetal fueron los siguientes:

Anomalías congénitas (polidactilia, labio leporino simple y microtia bilateral) 3 casos; ictericia habitual 3 casos, hemolítica 1 caso, por isoinmunización Rh 1 caso; prematurez 4 casos; infección 5 casos (neumonía aspirativa 1; lúes 1 ; conjuntivitis 1 ; enteritis 2 ); hidrocele 1 caso; erosión del cuero cabelludo por la ventosa de Malmstrom 1 caso.

Se encontraron 18 muertes fetales, 13 mortinatos y 5 neonatales, sobre los 261 fetos recibidos, para una incidencia de $2.7 \%$, que corregida desciende a $1.52 \%$.
En los mortinatos se encontró la siguiente patología asociada:

\begin{tabular}{lc}
\hline & Casos \\
\hline Antecedentes de toxemia & 3 \\
Nefropatía & 1 \\
Muerte habitual del feto & 1 \\
Hemorragia genital por & \\
$\quad$ placenta previa & 1 \\
Dos circulares al cuello & 1 \\
Segundo gemelo bivitelino & 1 \\
Aborto de 20 semanas & 1 \\
Prematurez con isoin- & \\
$\quad$ munización Rh & 1 \\
No se encontró dato & 3 \\
\hline
\end{tabular}


En los casos de muerte neonatal la causa fue la siguiente:

\section{Casos}

Prematurez y síndrome de dificultad respiratoria

Hemorragia cerebral en caso de parto en pelvis Isoinmunización por $\mathrm{Rh}$ Neumonía aspirativa Neumonía aspirativa e insuficiencia cardíaca

En nuestro trabajo anterior presentamos 8 casos de muerte fetal sobre 124 de parto vaginal postcesárea, para una incidencia de $6.45 \%$.

Riva y Teich (1) sobre 158 casos presentan 8 prematuros, de los cuales sobrevivieron el $37.5 \%$; la mortalidad fetal no corregida alcanzó al $2.8 \%$ y corregida fue nula.

Reyes Ceja (2) no presenta mortalidad en sus 190 casos.

Marcano y Agüero (3) presentan 57 muertes fetales sobre 424 gestaciones viables para una incidencia de $13.3 \%$, cifra realmente elevada; 37 fueron mortinatos y 20 pertenecen al período neonatal (13 prematuros) .

Jesurun y Simpson tienen una mortalidad perinatal del 5\%.

Del Corral y Muñoz (8) sobre 125 casos estudiados presentan 3 muertes fetales, dos de ellas en casos de ruptura uterina completa.

Cárdenas y Zornoza (10) en 60 casos de parto vaginal postcesárea presentan 1 mortinato y 3 muertes neona- tales correspondientes a un inmaduro y 2 prematuros.

Peralta Cayón (9) no presenta mortalidad fetal en su estudio.

\section{RESUMEN Y CONCLUSIONES}

1. Presentamos 614 casos de pacientes con antecedentes de cesárea, atendidas en el Instituto Materno Infantil de Bogotá, en un lapso de 19 meses.

Consideramos los resultados obtenidos en ellos, de acuerdo a normas precisas fijadas previamente en cuanto a valoración, conducción y conducta del parto, se refiere.

3. De los 614 casos estudiados, $282(45.9 \%)$ fueron considerados favorables para el parto vaginal, el cual se realizó en el $89.4 \%$, de ellos.

4. De los 614 casos $332(54.1 \%)$ fueron considerados desfavorables para parto vaginal, que solo se produjo en $21.1 \%$ de ellos.

5. Las causas más frecuentes de la primera cesárea fueron: placenta previa, sufrimiento fetal, situación transversa, procindencia del cordón y desproporción cefalo-pélvica.

6. Cerca del $80 \%$ de los casos correspondieron a embarazos a término, tanto por peso fetal como por edad gestacional.

7. La falta de control prenatal y el ingreso de las pacientes en trabajo de parto modifican el plan prefijado y disminuyen las posibilidades de mejorar los resultados materno-fetales. 
8. La mortalidad fetal perinatal, $50 \%$ de nuestros casos, porcentaje que no corregida, alcanzó el $7.2 \%$ cifra bien hubiéramos podido aumentar de inferior a la de otros centros que dis- no mediar las dificultades anteriormente ponen de mejores medios de atención. mencionadas.

Corregida alcanzó al $1.52 \%$.

9. Insistimos en la elevada frecuencia de distocias de contracción por defecto en las pacientes con antecedentes de cesárea; su incidencia alcanzó al $30 \%$ en los casos estudiados por nosotros.

10. Somos partidarios de prescindir del uso de soluciones de Pitocin endovenosamente en nuestro medio hospitalario, en el cual el elevado volumen de pacientes y la escasez de personal y de sistemas de control, constituyen un peligro no despreciable.

11. Aplicamos fórceps, espátulas o ventosa de Malmstrom en cerca del

12. Practicamos revisión uterina en cerca del $75 \%$ de nuestras pacientes.

13. Sólo un porcentaje b a j o de nuestros casos tuvieron trabajo prolongado (más de 12 horas); éste fue de $12.3 \%$.

14. Nuestra incidencia de ruptura uterina es baja (1.5\%) y los casos en los cuales se presentó corresponden a uso de Pitocin por vía intravenosa y a descuido en el control del trabajo, por lo cual los consideramos como prevenibles.

15. No tuvimos casos de mortalidad materna.

\section{BIBLIOGRAFIA}

1. RIVA Y TEICH: Vaginal delivery after cesarean section. A. J. O. V. 81, III/61.

2. REYES CEJA L.: La prueba de trabajo de parto en pacientes con cesárea anterior. Gin-Obst. de México. XIV de 1959.

4. MARCANO GUZMAN: Parto vaginal postcesárea. Revista de Obst-Gin. de Venezuela. XIX - 4/59.

5. PAEZ PUMAR: Cesárea iterada. Rev. de Obst-Gin. de Venezuela XIX. 4/59.

6. AgÜERo marcano y PAEZ: Desuniones de la herida uterina. Revista de ObsGin. de Venezuela. XIX. 4/59.

7. LAMKEE DONALsON: Cesarean sections. A. J. O. G. Vol. 83, № 5 de 1963.

8. MUÑOS y DEL CORRAL: Evaluación clínica para el parto después de cesárea. Rev. Colombiana de Obst-Gin-. X. 1 de 1959

9. PERALTA CAYON RAFAEL: Cesáreas previas y abortos. controversias clínicas y terapéuticas. 1962 .

10. CARDenas y zornosa: La cesárea iterativa: Rev. de la Clínica David Restrepo. Vol. III. 1 de 1962. 
11. SANZ ARAOS: Conducta obstétrica en pacientes previamente operadas de cesárea. Rev. Colombiana de Obs-Gin. III/52.

13. PILlSBURY S.: 'The safety of cesarean sections. A. J. O. G. Volumen 86 , No 5 de 1963.

14. ACUÑa GABRIEL: Parto espontáneo después de cesárea y cesáreá de repetición en el Instituto Materno Infantil. Revista Colombiana de Obst-Gin. XIII. 1. de 1962.

15. RABAGO JOSE: Conducta a seguir en las embarazadas con cesárea previa. GinObst. de México. XVI. 90, II/61.

16. SEPTIEN JOSE MANUEL: Conducta a seguir en las embarazadas con cesárea previa. Gin-Obst. de México. XVI. 90, II/61.

17. SUAREZ COBO MANUEL: Conducta a seguir en las embarazadas con cesárea previa. Gin-Obst. de México. XVI. 90. II/61.

19. CALCAÑo JULIO: Ruptura uterina en la Maternidad Concepción Palacios. ObstGin. de Venezuela. XIX de 1959.

20. Cosgrove roberto: Ruptura uterina. Rev. de obst-Gin. de México. XV. 127, de 1960 .

21. MacLenNAN A.: Fetal loss in cesarean sections. A. J. O. G. Volumen 82 № 1 de 1961. 